\title{
Humble and Kind: Cultural Humility as a Buffer of the Association between Social Dominance Orientation and Prejudice
}

\author{
Emilio Paolo Visintin ${ }^{1, *(1)}$ and Marika Rullo ${ }^{2}$ (I) \\ 1 Department of Humanities, University of Ferrara, 44121 Ferrara, Italy \\ 2 Department of Education, Human Sciences and Intercultural Communication, University of Siena, \\ 52100 Arezzo, Italy; marika.rullo@unisi.it \\ * Correspondence: emiliopaolo.visintin@unife.it
}

Citation: Visintin, E.P.; Rullo, M.

Humble and Kind: Cultural Humility as a Buffer of the Association between Social Dominance Orientation and Prejudice. Societies 2021, 11, 117. https://doi.org/10.3390/soc11040117

Academic Editors: Mancini Tiziana and Chiara Imperato

Received: 25 August 2021

Accepted: 20 September 2021

Published: 24 September 2021

Publisher's Note: MDPI stays neutral with regard to jurisdictional claims in published maps and institutional affiliations.

Copyright: (c) 2021 by the authors. Licensee MDPI, Basel, Switzerland. This article is an open access article distributed under the terms and conditions of the Creative Commons Attribution (CC BY) license (https:// creativecommons.org/licenses/by/ $4.0 /)$.

\begin{abstract}
With the rise of prejudice and discrimination against ethnic and immigrant minorities, strategies to reduce prejudice and discrimination, and to counteract the impact of intolerant, antiegalitarian ideologies, are needed. Here we focused on cultural humility, i.e., the ability to have a humble and other-oriented approach to others' cultural backgrounds, resulting from self-examination and critical thinking about structural privileges and inequalities. In this research we proposed that cultural humility might attenuate the effects of intolerant, anti-egalitarian ideologies such as social dominance orientation (SDO) and right-wing authoritarianism (RWA) on negative intergroup attitudes and perceptions. In a correlational study conducted in Italy, we found that cultural humility moderated the associations between SDO and prejudice toward immigrants, as well as between SDO and perceptions of threat posed by immigrants. Specifically, the associations of SDO with prejudice and threat were lower among respondents with high cultural humility compared to respondents with low cultural humility. Conversely, cultural humility did not moderate the effects of RWA on prejudice and threat. Findings are discussed considering the motivations underlying prejudice of high-SDO and high-RWA individuals, and proposing cultural humility training to foster positive intergroup relations.
\end{abstract}

Keywords: cultural humility; social dominance orientation; right-wing authoritarianism; prejudice; threat perceptions

\section{Introduction}

Despite growing ethnic and cultural diversity and opportunities for intergroup contact, current day societies are characterized by prejudice and discrimination against social minorities such as immigrants and refugees. These phenomena might be exacerbated by the rise of anti-immigration stances in political debate and by the ongoing economic and health crisis.

It is therefore crucial to identity factors which could contribute to promotion of positive intergroup perceptions and reduction in prejudice and discrimination. In this research, we focused on cultural humility, i.e., the ability to keep an other-oriented, humble approach when encountering people with a different cultural background [1]. While individuals differ in their cultural humility levels, cultural humility can also be successfully trained, e.g., [2,3]. Its role for the promotion of positive intergroup attitudes could therefore be crucial to reach a tolerant and egalitarian society.

In this research, we analyzed the possible role of cultural humility in buffering the associations between intolerant, anti-egalitarian ideologies and prejudice. Specifically, we considered right-wing authoritarianism (RWA), i.e., an ideological orientation consisting of strict adherence to conventional norms, submission to authorities, authoritarian aggression toward people and groups not following conventional norms and authorities [4], and 
social dominance orientation (SDO), i.e., the preference for hierarchical relations between groups [5]. In a correlational study conducted in Italy, we tested for the first time whether a culturally humble approach could moderate the associations between RWA and SDO on the one hand, and prejudice and threat perceptions toward immigrants in Italy on the other hand.

\subsection{Cultural Humility and Intergroup Relations}

Cultural humility, defined as the "ability to maintain an interpersonal stance that is other-oriented (or open to the other) in relation to aspects of cultural identity that are most important to the [person]" [1] (for a review see also [6]), is an emerging construct from the general domain of humility. The construct has been initially proposed in the context of the psychotherapeutic, educational, and health care professions [7-10], and has therefore been incorporated in multicultural competence training. More generally, cultural humility can be referred to as a "way of being" ([11], p. 214) aware of the impact of cultural differences in individuals' experiences and perspectives, including an awareness about privilege and systemic oppression and a recognition of social injustices incorporated into social structures $[12,13]$.

Although conceptualized as a way of being, a culturally humble mindset is the result of a lifelong learning process invoking critical reflection and self-examination [12]. In this sense, cultural humility might be conceived as an individual difference that can be developed and trained over the lifespan, e.g., $[2,3,14]$. The self-examination process leading to the development of cultural humility begins with the acknowledgment that one's cultural background is not superior as compared to others, and that encountering people with different cultural backgrounds and experiences could be a precious learning experience ([1], p. 353). Due to deep self-examination, people can challenge their beliefs and potential prejudices by questioning how systemic factors, such as power imbalances, have influenced them [11]. Consequently, a culturally humble perspective promotes respectful cross-cultural relationships and interactions $[1,15,16]$, characterized by an open stance toward new ideas and perspectives when engaging in challenging conversation with others.

Although most of the research on cultural humility, since the pioneering work by Tervalon and Murray-Garcia [17], has focused on health care, education, and psychotherapy, this construct has recently nurtured the attention of many researchers dealing with expressions of prejudice and discrimination across social groups. For instance, a recent study by Rullo et al. [18] conducted in Italy, found that cultural humility can buffer the effects of the antecedents of intergroup tensions, reducing the association between perceived diversity and negative intergroup contact. In their correlational study, the authors found that Italian participants with low cultural humility were inclined to experience opportunities for contact with immigrants and Muslims more negatively compared to people with high cultural humility, and consequently were inclined to express more prejudice toward immigrants and Muslims. At the same time, they also found that when people with low cultural humility engaged in positive contact with immigrants and Muslims, their expression of prejudice was reduced. These findings were in line with earlier studies showing that embodying cultural humility can foster positive intergroup relations, for instance by reducing the dehumanization of immigrants [19], reducing prejudice and discrimination toward Syrian refugees [20], or by increasing openness toward religious, ethnic, and sexual minorities $[6,9,13,21,22]$.

Thus, in line with previous findings, in the present research we proposed that cultural humility could contribute to positive intergroup relations by attenuating the effect of intolerant and anti-egalitarian ideologies on intergroup attitudes and perceptions.

\subsection{Social Dominance Orientation, Right-Wing Authoritarianism and Intergroup Relations}

Ideologies are considered to be strong antecedents of intergroup attitudes. Specifically, the roles of SDO and RWA on intergroup attitudes have received large attention. SDO is 
defined as the acceptance of and preference for intergroup hierarchies [5]. RWA, in turn, has been defined as the combination of three attitudes [4]: authoritarian submission, i.e., acritical acceptance of and obedience and submission to authorities perceived as legitimate; authoritarian aggression, i.e., aggression toward people and groups not adhering to conventional social norms and authorities; and conventionalism, i.e., strong acceptance and adherence to social norms and rejection of anything or anyone going against such norms.

Endorsement of both SDO and RWA is positively associated with prejudice toward different outgroups, especially minority outgroups, such as immigrants, ethnic minorities, and sexual minorities, e.g., [23,24]. While both ideologies are associated with intolerant attitudes toward social minorities, some differences in their associations with prejudice and negative intergroup behaviors emerge. Indeed, according to the dual process motivational approach [25], there are different motivations behind the prejudice of high-SDO people and of high-RWA people. Individuals with high-SDO perceive the world as a competitive jungle, endorse values such as dominance and power, and have a motivation to preserve status hierarchies. Individuals with high-RWA instead perceive the world as dangerous and feel threatened by outgroups not conforming to social norms or authority, and have a motivation to preserve conformity to norms and obedience to authorities. In line with the dual process motivational approach, Duckitt and Sibley [26] found that RWA was a strong predictor of prejudice against outgroups perceived as dangerous and threatening (e.g., terrorists), while SDO was a strong predictor of prejudice against outgroups perceived as low in status or challenging status hierarchies (e.g., unemployed people). In this vein, Thomsen, Green, and Sidanius [27] found that RWA elicited negative behavioral tendencies when primed with immigrants refusing to assimilate into the receiving society and therefore posing a threat to ingroup conformity, while SDO elicited negative behavioral tendencies when primed with immigrants willing to assimilate into the receiving society and therefore posing a threat to intergroup status boundaries.

Crucially for the current research, while both SDO and RWA have been consistently found to be associated with prejudice against immigrants, their effects might be shaped by other factors, and associations between such ideologies and prejudice might be attenuated. For example, personal experiences of intergroup contact could reduce associations between such ideologies and prejudice, e.g., [28,29] for RWA; [30] for SDO; and [31] for a review on the effects of intergroup contact among people endorsing intolerant ideologies. This mirrors the conceptualization of SDO in social dominance theory [32] which postulates that SDO and its effects might be shaped by personal, interpersonal, and intergroup experiences. Turning to RWA, Gorska et al. [33] found that the association between RWA and prejudice is reduced (or in some cases even reversed) in the presence of tolerant social norms. According to the authors, individuals with high-RWA are strongly motivated to adhere to social norms and might therefore reduce their prejudice expressions when social norms are tolerant.

Based on previous theorizations and research proposing that associations between intolerant ideologies and prejudice can be shaped by other variables, we tested whether the associations of SDO and RWA with intergroup attitudes and perceptions can be shaped by cultural humility.

\subsection{The Current Research}

This research was aimed at testing whether cultural humility could moderate the associations between intolerant, prejudice-enhancing ideologies and intergroup perceptions and attitudes. As previously explained, cultural humility is associated with positive intergroup attitudes and perceptions, e.g., [20,22], involves an awareness and critical thinking about structural privileges and injustices [11], and can curb the effects of antecedents on negative intergroup relations [18].

Therefore, we tested the hypotheses that cultural humility could moderate the associations between SDO and intergroup attitudes and perceptions (H1), and between RWA and intergroup attitudes and perceptions (H2). Specifically, we expected that the associations 
between SDO and negative intergroup attitudes and perceptions and between RWA and negative intergroup attitudes and perceptions could be reduced among people with high cultural humility.

These hypotheses were tested in a correlational study conducted with Italian participants reporting their cultural humility, SDO, and RWA, as well as their attitudes and perceptions toward immigrants in Italy. The hypothesized associations were tested on two outcome variables, i.e., prejudice assessed as negative (vs. positive) attitudes toward immigrants, and perceptions that immigrants pose a threat to the Italian economy, safety, culture, and identity [34].

\section{Materials and Methods}

\subsection{Data Collection and Participants}

Three research assistants enrolled in a bachelor program in Educational Sciences invited potential respondents from their social networks to answer an online questionnaire. All participants provided informed consent before answering the questionnaire. The research was designed following guidelines established by the Italian Psychological Association and by the American Psychological Association, and in accordance with the EU General Data Protection Regulation.

The questionnaire, or part thereof, was answered by 266 respondents. We excluded from the data analysis two respondents not living in Italy, two respondents younger than 18 , and one respondent who did not complete the questionnaire and who fully skipped the SDO and RWA items. The final sample included 261 respondents. All had Italian nationality and were living in Italy at the time of data collection. The age of respondents ranged between 19 and 71 years of age $(M=32.97, S D=14.80 ; 9$ missing data). $75 \%$ $(n=196)$ of respondents were female (one gender fluid and 2 missing data). The sample was diversified in terms of educational level, with $5.7 \%$ of respondents declaring lower secondary degree as their highest educational level, $49 \%$ of respondents with a higher secondary degree, and the remaining respondents having at least a bachelor's degree (2 missing data).

\subsection{Measures in the Questionnaire}

Cultural humility was assessed using an adaptation of the cultural humility scale [1]. To reduce social desirability, we adapted the procedure used by Schwartz et al. [35] to assess human values, and therefore invited respondents to rate how similar a person is to them on 12 items with a response scale ranging from 1 (not at all like me) to 4 (completely like me). Sample items were "Is genuinely interested in learning more about other cultures" and "Makes assumptions about other cultures" (reverse coded). The reliability of this measure was good (Cronbach's alpha $=0.75)$.

Social dominance orientation was assessed using the Short SDO scale by Pratto et al. [36] which was previously validated in the Italian context. Respondents were asked to rate their agreement on a 5 -point scale $(1=$ strongly disagree; $5=$ strongly agree $)$ with four items (e.g., "Superior groups should dominate inferior groups"). The reliability of this measure was modest (Cronbach's alpha $=0.58$ ). Given that the measure was validated, we retained the four items in the main analysis. However, given that excluding the first item ("In setting priorities, we must consider all groups", reverse coded) would improve the reliability (Cronbach's alpha $=0.66$ ), all data analysis were re-run with a three item SDO score excluding the first SDO item, and the results pattern did not change.

Right-wing authoritarianism was measured by asking respondents to rate their agreement ( 1 = strongly disagree; 5 = strongly agree $)$ with three items based on Altemeyer [4], which tap the three components of RWA: "We should take strong measures against deviants in society" for authoritarian aggression, "We need strong leaders to live safely in society" for authoritarian submission, and "Traditions should never be questioned" for conventionalism. The reliability of the three items was acceptable (Cronbach's alpha $=0.61$ ). 
Prejudice was assessed with a question asking respondents to rate their attitude toward immigrants on a scale from 0 (extremely unfavorable) to 10 (extremely favorable). Answers were reverse coded, so that higher scores represented more prejudice.

Threat was measured with six items adapted from Stephan et al. [34]. Respondents were asked to rate their agreement on a 4 -point scale $(1=$ fully disagree; $4=$ fully agree $)$ with items investigating the perception that immigrants are a threat to Italian values, identity, economy, and safety (e.g., "Italian identity is threatened by the presence of immigrants in Italy"). While the items referred to both symbolic (values and identity) and realistic (economy and safety) threats, a preliminary principal component analysis showed that the six items loaded onto a single dimension explaining $65 \%$ of variance (Eigenvalue $=3.88$; factor loadings $\geq 0.74$ ). This measure was reliable (Cronbach's alpha $=0.89)$.

The questionnaire also included additional measures. Details about the additional measures can be obtained upon request from the corresponding author. Among the additional measures, there were two items investigating the frequency of contact with immigrants [37], on a response scale from 1 (never) to 4 (very often). These two questions were: "How frequently do you have contact with immigrants?" and "How frequently do you voluntarily interact with immigrants?". This measure was reliable (Spearman-Brown statistic $\rho=0.63$ ). The frequency of intergroup contact was treated as a control variable in the additional analysis.

\section{Results}

\subsection{Preliminary Analysis}

Missing data on the measures in the questionnaire were $0.52 \%$ and were missing completely at random, as shown by a non-significant Little MCAR test, $\chi^{2}(385)=406.54$, $p=0.216$. Missing data were imputed using the EM algorithm in SPSS.

After reverse coding the answers to five cultural humility and two SDO items, for all multiple-item measures we created a composite score averaging the respective items.

To test the empirical distinction between variables, we ran a confirmatory factor analysis in Mplus. We used the partial disaggregation approach [38]. For cultural humility, SDO, RWA, and threat we created parcels (three parcels for cultural humility and threat, two parcels for SDO and RWA) by averaging subsets of items. Prejudice was instead assessed using a single item. A model with five latent variables and eleven observed variables fitted the data well, $\chi^{2}(35)=62.25, p=0.003, \chi^{2} / \mathrm{df}$ ratio $=1.78$, RMSEA $=0.055$, $\mathrm{SRMR}=0.027$, and CFI $=0.98$. All factor loadings were significant $(p<0.001)$ and $\geq 0.62$. The variables were empirically distinct, with all correlations between the latent variables lower than I 1 ( (95\% confidence interval).

Table 1 reports the means, standard deviations, and correlations between the variables. Cultural humility was relatively high, while SDO, prejudice, and threat were relatively low.

Table 1. Means, standard deviations, and correlations between variables.

\begin{tabular}{lccccc}
\hline & Mean (SD) & $\mathbf{1}$ & $\mathbf{2}$ & $\mathbf{3}$ & $\mathbf{4}$ \\
\hline 1. Cultural humility & $3.27(0.39)$ & - & & & \\
2. SDO & $1.86(0.73)$ & -0.44 & - & & \\
3. RWA & $2.76(1.02)$ & -0.27 & 0.23 & - & - \\
4. Prejudice & $2.93(2.30)$ & -0.51 & 0.52 & 0.27 & 0.71 \\
5. Threat & $1.75(0.70)$ & -0.50 & 0.56 & 0.46 & \\
\hline
\end{tabular}

Notes: All correlations are significant, $p<0.001$. SDO $=$ social dominance orientation. RWA = right-wing authoritarianism.

Cultural humility was negatively associated with SDO, RWA, prejudice, and threat. Conversely, both SDO and RWA were positively associated with prejudice and threat.

\subsection{Results}

To test whether cultural humility moderates the associations between SDO and intergroup attitudes and perceptions, and between RWA and intergroup attitudes and per- 
ceptions, we ran two regression analyses, one with prejudice and one with threat as the outcome variables. They were regressed on (centered) cultural humility, (centered) SDO, (centered) RWA, and the cultural humility $\times$ SDO and cultural humility $\times$ RWA interactions. The results are displayed in Table 2.

Table 2. Regression analysis predicting prejudice and threat.

\begin{tabular}{lcc}
\hline & Prejudice & Threat \\
\hline Intercept & $2.80(0.12)^{* * *}$ & $1.68(0.03)^{* * *}$ \\
Cultural humility & $-1.63(0.33)^{* * *}$ & $-0.33(0.09)^{* * *}$ \\
SDO & $1.08(0.17)^{* * *}$ & $0.33(0.05)^{* * *}$ \\
RWA & $0.22(0.11)^{\circ}$ & $0.21(0.03)^{* * *}$ \\
Cultural humility $\times$ SDO & $-1.41(0.38)^{* * *}$ & $-0.55(0.10)^{* * *}$ \\
Cultural humility RWA & $0.40(0.26)$ & $0.02(0.07)$ \\
$R^{2}$ & 0.41 & 0.53 \\
F & 35.72 & 58.50 \\
$d f$ & 5,255 & 5,255
\end{tabular}

Notes: Unstandardized coefficients (and standard errors) are reported. ${ }^{\circ} p=0.052 .{ }^{* * *} p<0.001 . \mathrm{SDO}=$ social dominance orientation. RWA = right-wing authoritarianism.

Cultural humility was negatively associated with both prejudice and threat, while both SDO and RWA were positively associated with prejudice and threat. The cultural humility $\times$ SDO interaction was significant both on prejudice and on threat $(\mathrm{H} 1)$, while the cultural humility $\times$ RWA interaction was not $(\mathrm{H} 2)$.

Decomposition of the interactions showed that SDO was associated with prejudice more strongly for respondents with low cultural humility $(-1 \mathrm{SD} ; B=1.62, S E=0.21$, $p<0.001)$ than for respondents with high cultural humility $(+1 \mathrm{SD} ; B=0.53, S E=0.24$, $p=0.026$ ) (see Figure 1). Similarly, SDO was associated with more threat for respondents with low cultural humility $(-1 \mathrm{SD} ; B=0.54, S E=0.06, p<0.001$ ), while the association between SDO and threat was not significant for respondents with high cultural humility (+1 SD; $B=0.12, S E=0.06, p=0.069)$ (see Figure 2).

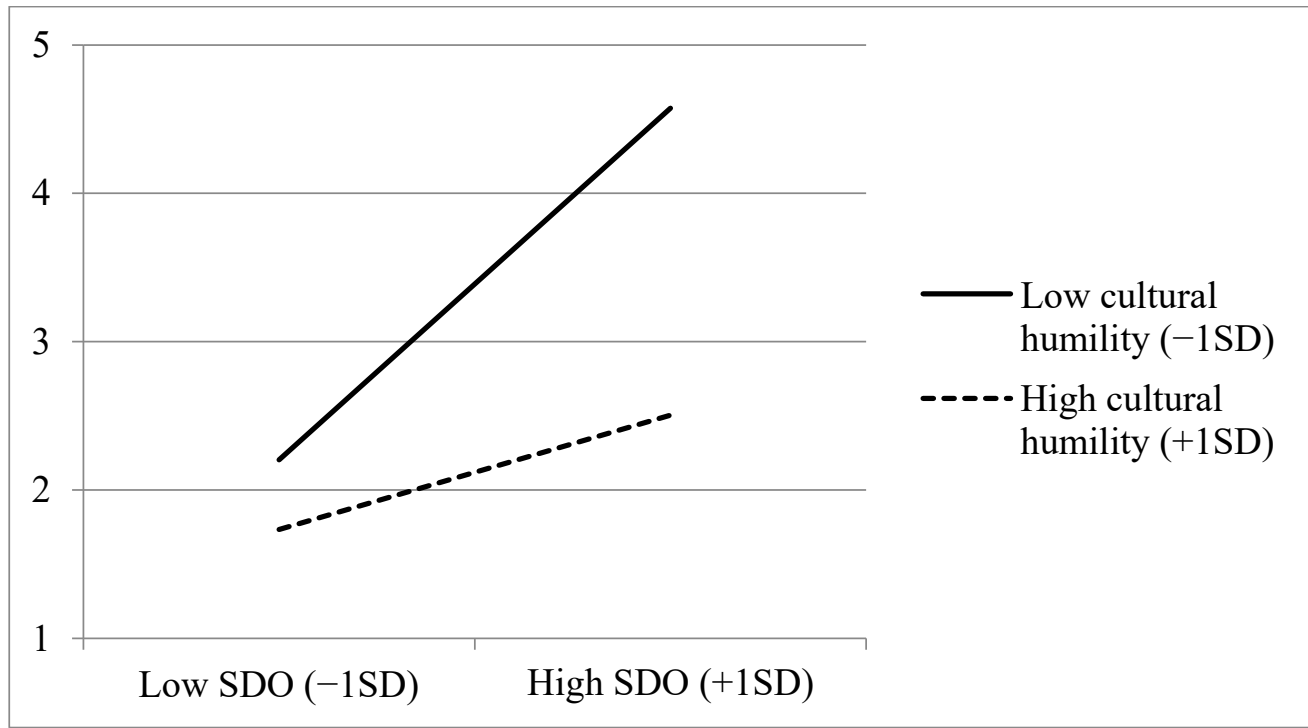

Figure 1. Prejudice as a function of social dominance orientation (SDO) and cultural humility. 


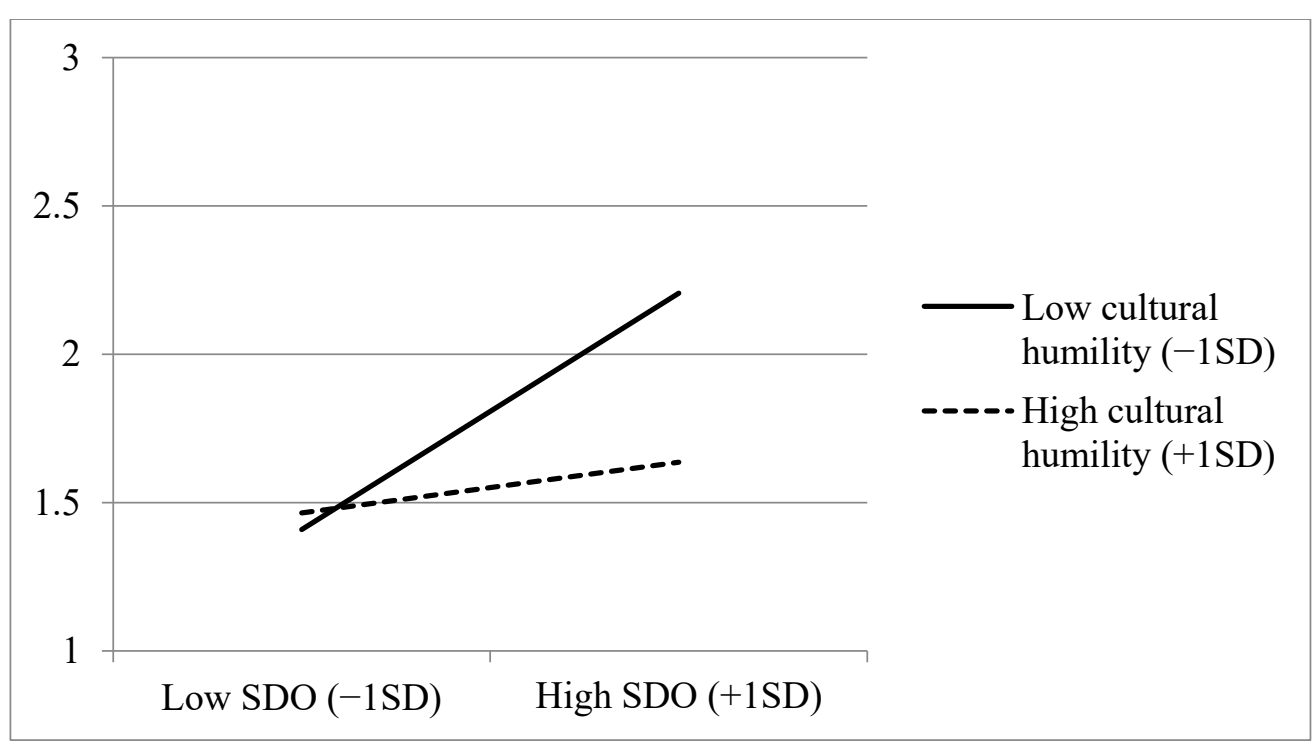

Figure 2. Perceived threat as a function of social dominance orientation (SDO) and cultural humility.

To check the robustness of these findings, the regression analyses were re-run controlling for intergroup contact, which covariates with both cultural humility [18] and with ideologies, e.g., [29], and for socio-demographic characteristics, i.e., gender, age, and educational level (gender was treated as dichotomous, i.e., male vs. female, and the participant who declared a genderfluid gender identity was not included in these additional analyses; missing data on socio-demographic characteristics were not imputed, therefore $n=248$ ). The results pattern did not change.

\section{Discussion}

In a correlational study conducted in Italy, we tested whether cultural humility could shape associations between SDO and RWA on the one hand, and prejudice toward immigrants and perceived threat posed by immigrants on the other hand. Supporting H1, we found that the associations of SDO with prejudice and with threat were lower among participants with high cultural humility compared to participants with low cultural humility. Conversely, cultural humility did not moderate associations between RWA and prejudice and threat, disconfirming $\mathrm{H} 2$.

Our research therefore supports the importance of a culturally humble approach for positive intergroup perceptions and attitudes. Indeed, cultural humility was not only associated with reduced prejudice and threat in line with previous research, e.g., [20,22], but it also buffered the effects of a strong predictor of negative intergroup attitudes and perceptions, i.e., SDO, on prejudice and threat (see also [18] for the role of cultural humility in reactions to perceived diversity). This research further confirms the conceptualization of SDO as a strong predictor of negative intergroup attitudes and perceptions, but whose effects can be attenuated by personal characteristics [32], namely, cultural humility.

But why did cultural humility moderate the effects of SDO but not of RWA on prejudice and threat? We believe that the explanation comes from some core facets of cultural humility and the different motivations behind the prejudice of high-SDO and high-RWA people. While high-RWA individuals endorse prejudice to preserve conformity to traditional social norms and obedience to authority, high-SDO individuals endorse prejudice to maintain and promote intergroup hierarchies [25]. A core facet of cultural humility is an awareness of and a critical thinking about structural privileges and injustices [11]. Therefore, cultural humility might be inconsistent with the desire to maintain and promote intergroup hierarchies, and might attenuate the impact of SDO on prejudice and negative perceptions. Instead, the reviewed definitions and conceptualizations of cultural humility do not deal with adherence to conventional norms and obedience to authority, and hence 
cultural humility might not have an impact on the motivations underlying prejudice of high-RWA people.

Despite the novel contributions in the literature on cultural humility and ideologies, some limitations of the current study need to be acknowledged. First, data were correlational and causality cannot be established. Future research could test the effectiveness of cultural humility training to counteract the effects of intolerant, anti-egalitarian ideologies on prejudice. Second, while we relied on a diversified sample in terms of age, gender, and educational level, the sample was a convenience sample recruited via three research assistants enrolled in an educational sciences bachelor program, and their research network might represent a more tolerant one compared to the general population. Future research could aim at replicating the present findings with representative samples. Third, we relied on self-report data, which might be biased because of social desirability. In line with the measurement of cultural humility proposed by Hook et al. [1], future research could employ external assessments of cultural humility. Outcome variables of the interplay between cultural humility and ideologies could include implicit prejudice or behavioral intentions and behaviors. Fourth, while the sample was homogeneous in terms of nationality (all respondents were Italian nationals), and while we controlled for gender, age, and educational level in the data analysis, we did not assess further participants' characteristics which could impact cultural humility, ideologies, and intergroup attitudes and perceptions. Future research could consider, for example, whether participants belong to an ethnic or religious minority or have an immigration background. Finally, this article is based on a single study conducted in Italy, with Italian national respondents and immigrants as the target outgroup. Future research could aim at replicating and generalizing our findings across other national and cultural contexts.

From an applied point of view, this research suggests the importance of cultural humility training in schools and educational contexts, healthcare contexts, and organizations [7-10]. Indeed, cultural humility training might contribute to the promotion of inclusive and egalitarian environments in the close context where cultural humility training takes place (e.g., schools, healthcare facilities, organizations), but also in societies in general. Closely linked to this research, cultural humility training could reduce the impact of anti-egalitarian ideologies and stances, which are spread within societies and are often promoted in political and mass-mediated debates.

Author Contributions: Conceptualization, E.P.V. and M.R.; methodology, E.P.V. and M.R.; formal analysis, E.P.V.; investigation, E.P.V.; data curation, E.P.V.; writing-original draft preparation, E.P.V. and M.R.; writing-review and editing, E.P.V. and M.R.; project administration, E.P.V. All authors have read and agreed to the published version of the manuscript.

Funding: This research received no external funding.

Institutional Review Board Statement: Our study did not involve clinical trials, deception, or experimental manipulations. Therefore, our universities' policies did not require us to have a formal approval for this study. Data collected were totally anonymous and analyzed in an aggregate manner, in compliance with Italian ethical standards, the European GPDR and the guidelines of the Declaration of Helsinki.

Informed Consent Statement: Informed consent was obtained from all participants involved in the study.

Data Availability Statement: Data can be retrieved at: https:/ / doi.org/10.6084/m9.figshare.166412 11.v2 (accessed on 23 September 2021).

Acknowledgments: The authors wish to thank Serena Balboni, Giulia Bonsi, and Elena Tiribello for their help with data collection.

Conflicts of Interest: The authors declare no conflict of interest. 


\section{References}

1. Hook, J.N.; Davis, D.E.; Owen, J.; Worthington, E.L., Jr.; Utsey, S.O. Cultural humility: Measuring openness to culturally diverse clients. J. Couns. Psychol. 2013, 60, 353-366. [CrossRef]

2. Isaacson, M. Clarifying concepts: Cultural humility or competency. J. Prof. Nurs. 2014, 30, 251-258. [CrossRef]

3. Juarez, J.A.; Marvel, K.; Brezinski, K.L.; Glazner, C.; Towbin, M.M.; Lawton, S. Bridging the gap: A curriculum to teach residents cultural humility. Fam. Med. 2006, 38, 97-102. [PubMed]

4. Altemeyer, B. Right-Wing Authoritarianism; University of Manitoba Press: Winnipeg, MB, Canada, 1981.

5. Sidanius, J.; Pratto, F. Social Dominance: An Intergroup Theory of Social Hierarchy and Oppression; Cambridge University Press: New York, NY, USA, 1999.

6. AlSheddi, M. Humility and Bridging Differences: A Systematic Literature Review of Humility in Relation to Diversity. Int. J. Intercult. Rel. 2020, 79, 36-45. [CrossRef]

7. Brown, E.L.; Vesely, C.K.; Dallman, L. Unpacking Biases: Developing Cultural Humility in Early Childhood and Elementary Teacher Candidates. Teach. Educ. J. 2016, 9, 75-96.

8. Chang, E.S.; Simon, M.; Dong, X. Integrating cultural humility into health care professional education and training. Adv. Health. Sci. Educ. 2012, 17, 269-278. [CrossRef]

9. Hook, J.N.; Farrell, J.E.; Johnson, K.A.; Van Tongeren, D.R.; Davis, D.E.; Aten, J.D. Intellectual humility and religious tolerance. J. Posit. Psychol. 2017, 12, 29-35. [CrossRef]

10. Kramlich, D.; Romano, A. Leveraging (the potential of) the multiethnic classroom: Using the constructs of cultural humility and safety to provide belonging for Cross Cultural Kid (CCK) refugees. Educ. Reflective Pract. 2020, 2020, 146-178. [CrossRef]

11. Foronda, C.; Baptiste, D.L.; Reinholdt, M.M.; Ousman, K. Cultural humility: A concept analysis. J. Transcult. Nurs. 2016, 27, 210-217. [CrossRef] [PubMed]

12. Fisher-Borne, M.; Cain, J.M.; Martin, S.L. From mastery to accountability: Cultural humility as an alternative to cultural competence. Soc. Work Educ. 2015, 34, 165-181. [CrossRef]

13. Mosher, D.K.; Hook, J.N.; Captari, L.E.; Davis, D.E.; DeBlaere, C.; Owen, J. Cultural humility: A therapeutic framework for engaging diverse clients. Pract. Innov. 2017, 2, 221-233. [CrossRef]

14. Schuessler, J.B.; Wilder, B.; Byrd, L.W. Reflective journaling and development of cultural humility in students. Nurs. Educ. Perspect. 2012, 33, 96-99. [CrossRef] [PubMed]

15. Drinane, J.M.; Owen, J.; Hook, J.N.; Davis, D.E.; Worthington, E.L., Jr. Microaggressions and cultural humility in psychotherapy. In Handbook of Humility: Theory, Research, and Applications; Worthington, E.L., Davis, D.E., Hook, J.N., Eds.; Routledge: New York, NY, USA, 2017; pp. 316-328.

16. Yeager, K.A.; Bauer-Wu, S. Cultural humility: Essential foundation for clinical researchers. Appl. Nurs. Res. 2013, 26, 251-256. [CrossRef]

17. Tervalon, M.; Murray-Garcia, J. Cultural humility versus cultural competence: A critical distinction in defining physician training outcomes in multicultural education. J. Health. Care Poor Underserved 1998, 9, 117-125. [CrossRef]

18. Rullo, M.; Visintin, E.P.; Milani, S.; Romano, A.; Fabbri, L. Stay humble and enjoy diversity: The interplay between intergroup contact and cultural humility on prejudice. Int. J. Intercult. Rel. 2021. under review.

19. Milani, S.; Rullo, M. Decostruire le differenze culturali: Una ricerca esplorativa sulle prospettive dei futuri educatori. Educ. Reflective Pract. 2020, 2020, 86-110.

20. Captari, L.E.; Shannonhouse, L.; Hook, J.N.; Aten, J.D.; Davis, E.B.; Davis, D.E.; Van Tongeren, D.; Hook, J.R. Prejudicial and welcoming attitudes toward Syrian refugees: The roles of cultural humility and moral foundations. J. Psychol. Theol. 2019, 47, 123-139. [CrossRef]

21. Choe, E.; Srisarajivakul, E.; Davis, D.E.; DeBlaere, C.; Van Tongeren, D.R.; Hook, J.N. Predicting attitudes towards lesbians and gay men: The effects of social conservatism, religious orientation, and cultural humility. J. Psychol. Theol. 2019, 47, 175-186. [CrossRef]

22. Van Tongeren, D.R.; Stafford, J.; Hook, J.N.; Green, J.D.; Davis, D.E.; Johnson, K.A. Humility attenuates negative attitudes and behaviors toward religious out-group members. J. Posit. Psychol. 2016, 11, 199-208. [CrossRef]

23. Mancini, T.; Bottura, B.; Caricati, L. The role of perception of threats, conservative beliefs and prejudice on prosocial behavioural intention in favour of asylum seekers in a sample of Italian adults. Curr. Psychol. 2020, 39, 2252-2261. [CrossRef]

24. Poteat, V.P.; Mereish, E.H. Ideology, prejudice, and attitudes toward sexual minority social policies and organizations. Polit. Psychol. 2012, 33, 211-224. [CrossRef]

25. Duckitt, J.; Sibley, C.G. A Dual-Process Motivational Model of Ideology, Politics, and Prejudice. Psychol. Inq. 2009, 20, 98-109. [CrossRef]

26. Duckitt, J.; Sibley, C.G. Right wing authoritarianism, social dominance orientation and the dimensions of generalized prejudice. Eur. J. Personal. 2007, 21, 113-130. [CrossRef]

27. Thomsen, L.; Green, E.G.; Sidanius, J. We will hunt them down: How social dominance orientation and right-wing authoritarianism fuel ethnic persecution of immigrants in fundamentally different ways. J. Exp. Soc. Psychol. 2008, 44, 1455-1464. [CrossRef]

28. Dhont, K.; Van Hiel, A. We must not be enemies: Interracial contact and the reduction of prejudice among authoritarians. Pers. Indiv. Differ. 2009, 46, 172-177. [CrossRef] 
29. Hodson, G.; Harry, H.; Mitchell, A. Independent benefits of contact and friendship on attitudes toward homosexuals among authoritarians and highly identified heterosexuals. Eur. J. Soc. Psychol. 2009, 39, 509-525. [CrossRef]

30. Visintin, E.P.; Berent, J.; Green, E.G.; Falomir-Pichastor, J.M. The interplay between social dominance orientation and intergroup contact in explaining support for multiculturalism. J. Appl. Soc. Psychol. 2019, 49, 319-327. [CrossRef]

31. Hodson, G. Do ideologically intolerant people benefit from intergroup contact? Curr. Dir. Psychol. Sci. 2011, $20,154-159$. [CrossRef]

32. Pratto, F.; Sidanius, J.; Levin, S. Social dominance theory and the dynamics of intergroup relations: Taking stock and looking forward. Eur. Rev. Soc. Psychol. 2006, 17, 271-320. [CrossRef]

33. Górska, P.; Stefaniak, A.; Lipowska, K.; Malinowska, K.; Skrodzka, M.; Marchlewska, M. Authoritarians Go with the Flow: Social Norms Moderate the Link between Right-Wing Authoritarianism and Outgroup-Directed Attitudes. Polit Psychol. 2021. [CrossRef]

34. Stephan, W.G.; Boniecki, K.A.; Ybarra, O.; Bettencourt, A.; Ervin, K.S.; Jackson, L.A.; McNatt, P.S.; Renfro, C.L. The role of threats in the racial attitudes of Blacks and Whites. Pers. Soc. Psychol. B. 2002, 28, 1242-1254. [CrossRef]

35. Schwartz, S.H.; Melech, G.; Lehmann, A.; Burgess, S.; Harris, M.; Owens, V. Extending the cross-cultural validity of the theory of basic human values with a different method of measurement. J. Cross. Cult. Psychol. 2001, 32, 519-542. [CrossRef]

36. Pratto, F.; Cidam, A.; Stewart, A.L.; Zeineddine, F.B.; Aranda, M.; Aiello, A.; Chryssochoou, X.; Cichocka, A.; Cohrs, J.C.; Durrheim, K.; et al. Social dominance in context and in individuals: Contextual moderation of robust effects of social dominance orientation in 15 languages and 20 countries. Soc. Psychol. Pers. Sci. 2013, 4, 587-599. [CrossRef]

37. Husnu, S.; Crisp, R.J. Elaboration enhances the imagined contact effect. J. Exp. Soc. Psychol. 2010, 46, 943-950. [CrossRef]

38. Little, T.D.; Cunningham, W.A.; Shahar, G.; Widaman, K.F. To parcel or not to parcel: Exploring the question, weighting the merits. Struct. Equ. Modeling 2002, 9, 151-175. [CrossRef] 